\title{
Cooperative Training and Latent Space Data Augmentation for Robust Medical Image Segmentation
}

\author{
Chen Chen $^{1}(\bowtie)$, Kerstin Hammernik ${ }^{1,2}$, Cheng Ouyang ${ }^{1}$, Chen Qin ${ }^{3}$, Wenjia \\ Bai $^{4,5}$, Daniel Rueckert ${ }^{1,2}$ \\ 1 BioMedIA Group, Department of Computing, Imperial College London, UK \\ 2 Klinikum rechts der Isar, Technical University of Munich, Germany \\ 3 Institute for Digital Communications, University of Edinburgh, UK \\ 4 Data Science Institute, Imperial College London, UK \\ 5 Department of Brain Sciences, Imperial College London, UK \\ chen. chen15@imperial.ac.uk
}

N

\begin{abstract}
Deep learning-based segmentation methods are vulnerable to unforeseen data distribution shifts during deployment, e.g. change of image appearances or contrasts caused by different scanners, unexpected imaging artifacts etc. In this paper, we present a cooperative framework for training image segmentation models and a latent space augmentation method for generating hard examples. Both contributions improve model generalization and robustness with limited data. The cooperative training framework consists of a fast-thinking network (FTN) and a slow-thinking network (STN). The FTN learns decoupled image features and shape features for image reconstruction and segmentation tasks. The STN learns shape priors for segmentation correction and refinement. The two networks are trained in a cooperative manner. The latent space augmentation generates challenging examples for training by masking the decoupled latent space in both channel-wise and spatial-wise manners. We performed extensive experiments on public cardiac imaging datasets. Using only 10 subjects from a single site for training, we demonstrated improved cross-site segmentation performance, and increased robustness against various unforeseen imaging artifacts compared to strong baseline methods. Particularly, cooperative training with latent space data augmentation yields $15 \%$ improvement in terms of average Dice score when compared to a standard training method.
\end{abstract}

\section{Introduction}

Segmenting anatomical structures from medical images is an important step for diagnosis, treatment planning and clinical research. In recent years, deep convolutional neural networks (CNNs) have been widely adopted to automate the segmentation procedure [1]2]. However, a major obstacle for deploying deep learning-based methods to real-world applications is domain shift during clinical deployment, which includes changes of image appearance and contrasts 
across medical centers and scanners as well as various imaging artefacts. Recent works on domain generalization provide a promising direction to address this issue $3,4,5,6,7$. A majority of them require training data from multiple domains to learn domain-invariant features for segmentation. Multi-domain datasets, however, may not always be feasible due to data privacy concerns and collection costs. Learning robust networks from single-domain data and limited data is of great practical value for medical imaging research.

In this work, we propose a novel cooperative training framework for learning a robust segmentation network from single-domain data. We make the following contributions. (1) First, to improve model performance on unseen domains, we design a cooperative training framework where two networks collaborate in both training and testing. This is inspired by the two-system model in human behaviour sciences [8], where a fast-thinking system makes intuitive judgment and a slow-thinking system corrects it with logical inference. Such a collaboration is essential for humans to deal with unfamiliar situations. In our framework, a fastthinking network (FTN) aims to understand the context of images and extracts task-related image and shape features for an initial segmentation. Subsequently, a slow-thinking network (STN) refines the initial segmentation according to a learned shape prior. (2) We introduce a latent space data augmentation (DA) method, which performs channel-wise and spacial-wise masking for the latent code learnt from FTN in random and targeted fashions. Reconstructing images with masked latent codes generates a diverse set of challenging images and corrupted segmentation maps to reinforce the training of both networks. Experimental results on cardiac imaging datasets show the cooperative training mechanism with generated challenging examples can effectively enhance FTN's segmentation capacity and STN's shape correction ability, leading to more robust segmentation. (3) The proposed method alleviates the need for multi-domain data and expertise for data augmentation, making it applicable to a wide range of applications.

Related Work. Our work is conceptually related to DA, multi-task learning (MTL). a) DA applies transformations or perturbations to improve the diversity of training data, which is effective for improving model generalization [9]. A large number of the works focuses on image-space DA, including both intensity and geometric transformation functions 10,11 and patch-wise perturbations $12,13,14,15$. Adversarial DA has also been explored, which takes the segmentation network into account and generates adversarial examples that can fool the network 16, 17, 18, 19. A major novelty of our work is that we perform DA in the latent space. The latent space contains abstract representation of both image and shape features and challenging examples can be generated by manipulating this space. Different from existing latent DA methods used in metric learning [20], our method is based on feature masking rather than feature interpolation and thus does not require paired images from the same/different categories to generate synthetic data. To the best of our knowledge, our work is the first to explore latent space DA for robust segmentation with single domain data. b) $M T L$ is extremely beneficial when training data is limited [14, 15, 21]. MTL 


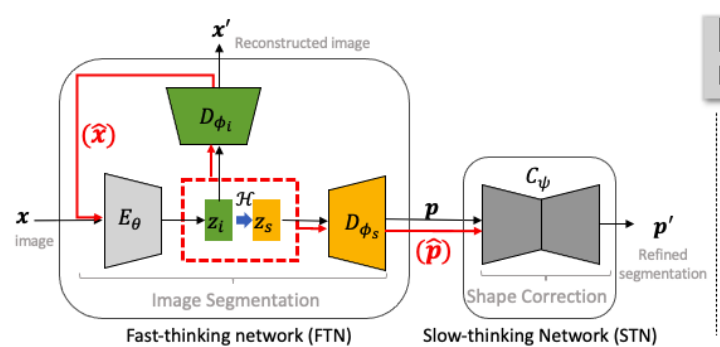

(a) Overview

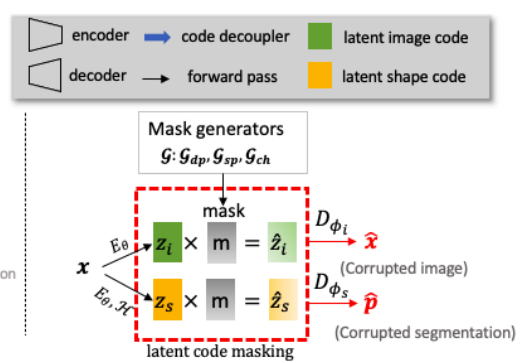

(b) Hard example generation

Fig. 1: (a) The proposed cooperative training framework, which consists of a fastthinking network (FTN) and a slow-thinking network (STN). (b) Hard example generation in latent space. Latent code masking is performed for generating both corrupted images and segmentations for cooperative training.

enhances network capacity by encouraging the learning of common semantic features across various tasks. Several methods consist of two stages of segmentation: a first network for coarse segmentation from images and a second network for refinement, where two networks are trained independently 22, 23. For example, in 22, manually designed functions are used to generate poor segmentation and a denoising autoencoder is independently trained for segmentation refinement. Another novelty of our work is that we seek the mutual benefits of a segmentation network and a denoising network by training them cooperatively, using hard examples constructed from latent space.

\section{Methodology}

Given a training dataset from one, single domain $D_{t r}=\left\{\left(\mathbf{x}_{i}, \mathbf{y}_{i}\right)\right\}_{i=1}^{n}$, with pairs of images $\mathbf{x}_{i} \in \mathbb{R}^{H \times W}$ and one-hot encoded $C$-class label maps $\mathbf{y}_{i} \in$ $\{0,1\}^{H \times W \times C}$ as ground truth (GT), our goal is to learn a robust segmentation network across various 'unseen' domains with different image appearance and/or quality. Here, $H, W$ denote image height and width, respectively.

\subsection{Overview of the framework}

An overview of the proposed framework is illustrated in Fig.11(a). At a high level, our framework consists of a fast-thinking network (FTN) and a slow-thinking network (STN). Given an image $\mathbf{x}$, the FTN extracts task-specific shape features $\mathbf{z}_{s}$ to perform the segmentation task and image contextual features $\mathbf{z}_{i}$ to perform the image reconstruction task. This network consists of a shared encoder $E_{\theta}$, a feature decoupler $\mathcal{H}$ and two task-specific decoders $\mathcal{D}_{\phi_{s}}$ and $\mathcal{D}_{\phi_{i}}$ for image segmentation and reconstruction tasks. We apply the latent code decoupler $\mathcal{H}$ to $\mathbf{z}_{i}$, so that task-unrelated information (e.g. image texture information, brightness) is deactivated in $\mathbf{z}_{s}$. This encourages a sparse latent code $\mathbf{z}_{s}$, which is beneficial 
for model robustness 24]. $\mathcal{H}$ employs a stack of two convolutional layers followed by a ReLU activation function. Please find Supple. Fig.5 for more details. STN is a denoising autoencoder network $\mathcal{C}_{\psi}$, which corrects the segmentation predicted by FTN by using a learned shape prior encoded in $\mathcal{C}_{\psi}$. At inference time, we first employ FTN to perform fast segmentation for a given image $\mathbf{x}$ : $\mathbf{p}=\mathcal{D}_{\phi_{s}}\left(\mathcal{H}\left(E_{\theta}(\mathbf{x})\right)\right)$, and then STN to refine the prediction for improved segmentation quality: $\mathbf{p}^{\prime}=\mathcal{C}_{\psi}(\mathbf{p})$.

\section{$2.2 \quad$ Standard training}

To train the two networks, we propose a standard approach which jointly trains the three encoder-decoder pairs with a supervised multi-task loss function for image reconstruction $\mathcal{L}_{\text {rec }}$, image segmentation $\mathcal{L}_{\text {seg }}$ and shape correction $\mathcal{L}_{\text {shp }}$. The loss is defined as:

$$
\mathcal{L}_{\text {std }}=\mathbb{E}_{(\mathbf{x}, \mathbf{y}) \in D_{t r}}\left[\mathcal{L}_{\text {rec }}\left(\mathbf{x}^{\prime}, \mathbf{x}\right)+\mathcal{L}_{\text {seg }}(\mathbf{p}, \mathbf{y})+\mathcal{L}_{\text {shp }}\left(\mathbf{p}^{\prime}, \mathbf{y}\right)+\mathcal{L}_{\text {shp }}\left(\mathbf{y}^{\prime}, \mathbf{y}\right)\right],
$$

where $\mathcal{L}_{\text {rec }}$ is the mean squared error (MSE) between the original input image $\mathbf{x}$ and the reconstructed image $\mathbf{x}^{\prime}=\mathcal{D}_{\phi_{i}}\left(E_{\theta}(\mathbf{x})\right), \mathcal{L}_{\text {seg }}$ and $\mathcal{L}_{\text {shp }}$ are crossentropy loss functions between ground truth $\mathbf{y}$ and predicted segmentation. The predicted segmentation can be the initial prediction $\mathbf{p}=\mathcal{D}_{\phi_{s}}\left(\mathcal{H}\left(E_{\theta}(\mathbf{x})\right)\right)$, or the reconstructed prediction $\mathbf{p}^{\prime}=\mathcal{C}_{\psi}(\mathbf{p})$ or reconstructed ground-truth map $\mathbf{y}^{\prime}=\mathcal{C}_{\psi}(\mathbf{y})$. Different from $\mathcal{L}_{\text {seg }}$, optimizing $\mathcal{L}_{\text {shp }}\left(\mathbf{p}^{\prime}, \mathbf{y}\right)$ will trigger gradient flows from STN to FTN. This allows STN to transfer shape knowledge to FTN to improve model generalizability.

\subsection{Latent space data augmentation for hard example generation}

Standard training is likely to suffer from over-fitting when training data is limited. To solve this problem, a novel latent space DA method is proposed which allows FTN to automatically construct hard examples. As shown in Fig. 1(b), the proposed method requires a mask generator $\mathcal{G}$ to produce a mask $\mathbf{m}$ on the latent code $\mathbf{z}$. The masked latent code $\hat{\mathbf{z}}=\mathbf{z} \cdot \mathbf{m}$ is then fed to the decoders to reconstruct a corrupted image $\hat{\mathbf{x}}=\mathcal{D}_{\phi_{i}}\left(\hat{\mathbf{z}}_{i}\right)$ and segmentation $\hat{\mathbf{p}}=\mathcal{D}_{\phi_{s}}\left(\hat{\mathbf{z}}_{s}\right)$. Here, - denotes element-wise multiplication. In our work, we use latent code masking for data augmentation. This differs from existing latent code dropout techniques for explicit regularization 25,26]. By dynamically masking the latent code, the proposed method can generate samples with a wide diversity of image appearances and segmentations, which are not bound to specific image transformation or corruption functions. Below we introduce three latent-code masking schemes: random dropout $\mathcal{G}_{d p}$, and two targeted masking schemes, channel-wise targeted mask generation $\mathcal{G}_{c h}$ and spatial-wise targeted mask generation $\mathcal{G}_{s p}$.

(1) Random Masking with Dropout A naïve approach for latent code masking is random channel-wise dropout [26, which is an enhanced version of the original dropout method. An entire channel of the latent code can be masked 
with all zeros at a probability of $p$ at training. Mathematically, this can be viewed as sampling a mask from a Bernoulli distribution:

$$
\mathcal{G}_{d p}\left(\mathbf{m}^{(i)} ; p\right)=\left\{\begin{array}{rl}
p \quad \mathbf{m}^{(i)} & =\mathbf{0} \in \mathbb{R}^{h \times w} \\
1-p \mathbf{m}^{(i)}=\mathbf{1} \in \mathbb{R}^{h \times w} ;
\end{array} \quad \forall i \in 1, \ldots, c .\right.
$$

The masked code at $i$-th channel is obtained via $\hat{\mathbf{z}}^{(i)}=\mathbf{z}^{(i)} \cdot \mathbf{m}^{(i)}$. In the following, we will use i-j-k to denote the three coordinates of latent code $\mathbf{z} \in \mathbb{R}^{c \times h \times w}$.

(2) Targeted Masking Inspired by the recent success on latent code masking for domain generalized image classification algorithm 25, we propose targeted latent code masking schemes which takes gradients as a clue to identify 'salient' features to mask. Following the common practice in adversarial DA [27, 28], we take task-specific losses (image reconstruction loss and image segmentation loss) to calculate the gradients $\mathbf{g}_{\mathbf{z}_{i}}, \mathbf{g}_{\mathbf{z}_{s}}$ for $\mathbf{z}_{i}$ and $\mathbf{z}_{s}$ respectively, formulated as: $\mathbf{g}_{\mathbf{z}_{i}}=$ $\nabla_{\mathbf{z}_{i}} \mathcal{L}_{\text {rec }}\left(\mathcal{D}_{\phi_{i}}\left(\mathbf{z}_{i}\right), \mathbf{x}\right), \mathbf{g}_{\mathbf{z}_{s}}=\nabla_{\mathbf{z}_{s}} \mathcal{L}_{\text {seg }}\left(\mathcal{D}_{\phi_{s}}\left(\mathbf{z}_{s}\right), \mathbf{y}\right)$. By ranking the values of taskspecific gradients, we can identify most predictive elements in the latent space to attack. We hypothesize that the elements with high response to task-specific loss functions are leading causes to performance drop under unforeseen domain shifts. We therefore focus on attacking these primary elements to simulate strong data distribution shifts. Two types of targeted masking are implemented, which mask features in latent code $\mathbf{z}$ along the channel dimension and spatial dimension.

a) channel-wise mask generator:

$$
\mathcal{G}_{c h}\left(\mathbf{m}^{(i)} ; \mathbf{g}_{\mathbf{z}}, p\right)=\left\{\begin{array}{l}
\mathbf{m}^{(i)}=a \mathbf{1} \in \mathbb{R}^{h \times w} \text { if } \mathbb{E}\left[\mathbf{g}_{\mathbf{z}}^{(i)}\right] \geq z_{p}^{c h} \\
\mathbf{m}^{(i)}=\mathbf{1} \in \mathbb{R}^{h \times w} \text { if } \mathbb{E}\left[\mathbf{g}_{\mathbf{z}}^{(i)}\right]<z_{p}^{c h} ;
\end{array} \forall i \in 1, \ldots, c,\right.
$$

b) spatial-wise mask generator:

$$
\mathcal{G}_{s p}\left(\mathbf{m}^{(j, k)} ; \mathbf{g}_{\mathbf{z}}, p\right)=\left\{\begin{array}{l}
\mathbf{m}^{(j, k)}=a \mathbf{1} \in \mathbb{R}^{c} \text { if } \mathbb{E}\left[\mathbf{g}_{\mathbf{z}}^{(j, k)}\right] \geq z_{p}^{s p} \\
\mathbf{m}^{(j, k)}=\mathbf{1} \in \mathbb{R}^{c} \text { if } \mathbb{E}\left[\mathbf{g}_{\mathbf{z}}^{(j, k)}\right]<z_{p}^{s p} ;
\end{array} \forall j \in[1, h], \forall k \in[1, w] .\right.
$$

Thresholds $z_{p}^{c h}, z_{p}^{s p} \in \mathbb{R}$ are top $p$-th value across the channel means and spatial means. $a$ is an annealing factor randomly sampled from $(0,0.5)$ to create soft masks. Compared to hard-masking $(a=0)$, soft-masking generates more diverse corrupted data (Supple. Fig.1). Channel-wise masked code at $i$-th channel is obtained via $\hat{\mathbf{z}}^{(i)}=\mathbf{z}^{(i)} \cdot \mathbf{m}^{(i)}$. Spatial-wise masked code at $(j, k)$ position is obtained via $\hat{\mathbf{z}}^{(j, k)}=\mathbf{z}^{(j, k)} \cdot \mathbf{m}^{(j, k)}$.

\subsection{Cooperative training}

During training, we randomly apply one of the three mask generators to both $\mathbf{z}_{i}, \mathbf{z}_{s}$. This process generates a rich set of corrupted images $\hat{\mathbf{x}}$ and segmentations $\hat{\mathbf{p}}$ on-the-fly. It allows us to train our dual-network on three hard example pairs, i.e. corrupted images-clean images $(\hat{\mathbf{x}}, \mathbf{x})$, corrupted images-GT $(\hat{\mathbf{x}}, \mathbf{y})$, corrupted prediction-GT $(\hat{\mathbf{p}}, \mathbf{y})$. The final loss for the proposed cooperative training method is a combination of losses defined on easy examples and hard examples: $\mathcal{L}_{\text {cooperative }}=\mathcal{L}_{\text {std }}+\mathcal{L}_{\text {hard }}$, where $\mathcal{L}_{\text {hard }}$ is defined as:

$$
\mathcal{L}_{\text {hard }}=\mathbb{E}_{\hat{\mathbf{x}}, \hat{\mathbf{p}}, \mathbf{x}, \mathbf{y}}\left[\mathcal{L}_{\text {rec }}\left(\mathcal{D}_{\phi_{i}}\left(E_{\theta}(\hat{\mathbf{x}})\right), \mathbf{x}\right)+\mathcal{L}_{\text {seg }}(\overline{\mathbf{p}}, \mathbf{y})+\mathcal{L}_{\text {shp }}\left(\mathcal{C}_{\psi}(\hat{\mathbf{p}}), \mathbf{y}\right)+\mathcal{L}_{s h p}\left(\mathcal{C}_{\psi}(\overline{\mathbf{p}}), \mathbf{y}\right)\right] .
$$

Here, $\overline{\mathbf{p}}=\mathcal{D}_{\phi_{i}}\left(\mathcal{H}\left(E_{\theta}(\hat{\mathbf{x}})\right)\right)$ is FTN's predicted segmentation on $\hat{\mathbf{x}}$. 


\section{Experiments and Results}

Datasets. To evaluate the efficacy of the proposed method, we apply it to the cardiac image segmentation task to segment the left ventricle cavity, left ventricular myocardium and right ventricle from MR images. Three datasets are used, ACDC [29, M\&M:730 and corrupted ACDC, named as ACDC-C. For all experiments, the training set is a single-site set of only 10 subjects from ACDC. 10 and 20 subjects from ACDC are used for validation and intra-domain test. The multi-site M\&Ms dataset (150 subjects from 5 different sites) is used for cross-domain test. The ACDC-C dataset is used for evaluating the robustness of the method for corrupted images. Challenging scenarios are simulated, where 20 ACDC test subjects are augmented three times with four different types of MR artefacts: bias field, ghosting, motion and spike artifacts 31 using the TorchIO ${ }^{8}$ toolkit. This produces 4 subsets with 60 subjects, named as RandBias, RandGhosting, RandMotion, RandSpike in experiments.

Implementation and evaluation. We employed the image pre-processing and default DA pipeline described in 17, including common photo-metric and geometric image transformations. Our encoder and decoder pairs support general structures. Without loss of generality, we used a U-net like structure [32]. Supple. Fig.5 visualizes detailed structures of encoder-decoder pairs as well as the latent space decoupler. For mask generation, we randomly select one type of proposed masking schemes at training, where $p$ is randomly selected from $[0 \%, 50 \%]$. We use the Adam optimizer with a batch size of 20 to update network parameters, with a learning rate $=1 e^{-4}$. Our code will be available on the Github? For all methods, we trained the same network three times using a set of randomly selected 10 ACDC subjects (600 epochs each run, on an Nvidia ${ }^{\circledR}$, using Pytorch). The average Dice score is reported for segmentation performance evaluation.

Experiment 1: Standard training vs cooperative training. We compared the proposed cooperative training method with the standard training method (using $\mathcal{L}_{\text {standard }}$ only) using the same backbone network structure. Fig. 2 shows the box-plots for each method. While both methods achieve comparable performance on the intra-domain test set (p-value $>0.1$ ), it is clear that cooperative training with dual-network $(\mathrm{FTN}+\mathrm{STN})$ yields the best performance across out-of domain test sets (see dark green boxes). Consistent improvements made by STN can be clearly observed across all domains. By contrast, STN with standard training fails to provide significant improvements on some datasets (pvalue $>0.1$ ). This indicates the superiority of cooperative training with latent space DA.

6 https://www.creatis.insa-lyon.fr/Challenge/acdc/databases.html

7 https://www.ub.edu/mnms/

8 https://github.com/fepegar/torchio

9 https://github.com/cherise215/Cooperative_Training_and_Latent_Space_Data_ Augmentation 


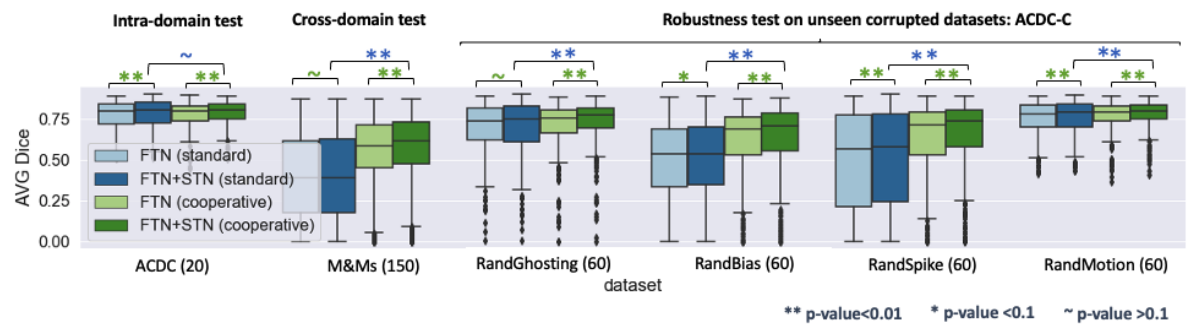

Fig. 2: Compared to standard training, cooperative training with self-generating hard examples greatly improves the segmentation performance on various unseen, challenging domains ( $\mathrm{p}$-value $<0.01$, average improvement: $15 \%$ ).

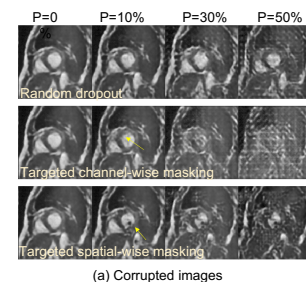

(a) Corrupted images

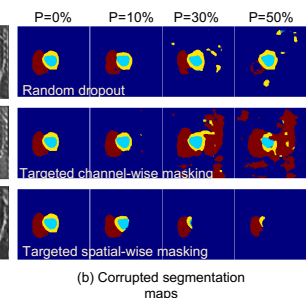

maps

Fig. 3: Visualization of generated hard examples with the three masking schemes ( $a=0$ for ease of comparison). Best viewed in zoom-in.

Experiment 2: Latent space DA vs Image space DA. We compared the proposed latent space -based method to other competitive image space DA methods: a) random multi-window in-and-out masking (Rand MWM) 14, 15, which uses an enhanced variant of Cutout [34] and Patch Gaussian 13 to introduce patch-wise perturbation to images; b) random convolutional kernels (Rand Conv) [33], which applies various random convolutional kernels to augment image texture and appearance variations; c) adversarial noise (Adv Noise) 16]; d) adversarial bias field (Adv Bias) [17, which augments image styles by adding realistic intensity inhomogeneities. We visualize augmented images using above methods in Supple. Fig.2 for better illustration. For methods under comparison, we used their official code implementation if available and ran experiments using the same backbone network for fairness. Results are shown in Table 1 .

Surprisingly, with limited training data, both random and adversarial DA methods do not necessarily improve the network generalization on all datasets. While AdvBias achieves the best performance on $M \& M s$ dataset and RandBias, this method has a side effect, making it more sensitive to the spiking artifacts (Dice score 0.4901 vs 0.3840). By contrast, the proposed latent space DA achieves the top average performance across six datasets, without any dramatic failures (Dice score $<0.5$ ). Similar results can be found in a large training setting, see Supple. Fig.3. Our method can generate not only perturbed images but also re- 
Table 1: Comparison to image space data augmentation methods for domain generalization. The proposed latent space augmentation method improves the performance on out-of-domain datasets compared to image space data augmentation methods. AVG: Average Dice scores across six datasets.

\begin{tabular}{lccccccc|c}
\hline Method & \multicolumn{3}{c}{ ACDC } & M\&Ms RandBias RandGhosting RandMotion RandSpike & $\begin{array}{c}\text { AVG } \\
(\text { FTN })\end{array}$ & $\begin{array}{c}\text { AVG } \\
(\text { FTN+STN) }\end{array}$ \\
\hline Standard training & 0.7681 & 0.3909 & 0.4889 & 0.6964 & 0.7494 & 0.4901 & 0.5970 & 0.6018 \\
\hline Rand MWM [14] & 0.7515 & 0.3984 & 0.4914 & 0.6685 & 0.7336 & 0.5713 & 0.6024 & 0.6131 \\
Rand Conv [33] & 0.7604 & 0.4544 & 0.5538 & 0.6891 & 0.7493 & 0.4902 & 0.6162 & 0.6404 \\
Adv Noise 16] & 0.7678 & 0.3873 & 0.4903 & 0.6829 & 0.7543 & 0.6244 & 0.6178 & 0.6276 \\
Adv Bias [17] & 0.7573 & $\mathbf{0 . 6 0 1 3}$ & $\mathbf{0 . 6 7 0 9}$ & 0.6773 & 0.7348 & 0.3840 & 0.6376 & 0.6604 \\
\hline Proposed w. $\hat{\mathbf{x}}$ & 0.7497 & 0.5154 & 0.5921 & 0.6921 & 0.7417 & $\mathbf{0 . 6 6 3 3}$ & 0.6591 & 0.6709 \\
Proposed w. $\hat{\mathbf{x}}, \hat{\mathbf{p}}$ & $\mathbf{0 . 7 6 9 6}$ & 0.5454 & 0.6174 & $\mathbf{0 . 7 0 7 3}$ & $\mathbf{0 . 7 6 4 3}$ & 0.6226 & $\mathbf{0 . 6 7 1 1}$ & $\mathbf{0 . 6 9 0 1}$ \\
\hline
\end{tabular}

Table 2: Effectiveness of targeted masking, latent code decoupler $\mathcal{H}$ and cooperative training

\begin{tabular}{|c|c|c|}
\hline Methods & FTN & $\mathrm{FTN}+\mathrm{STN}$ \\
\hline w.o. $\mathcal{G}_{c h}, \mathcal{G}_{s p}$ & 0.6344 & 0.6584 \\
\hline $\begin{array}{l}\text { share code (a) }\left(\mathbf{z}_{i}=\mathbf{z}_{i}, \mathbf{z}_{s}=\mathbf{z}_{i}\right) \\
\text { share code (b) }\left(\mathbf{z}_{i}=\mathbf{z}_{s}, \mathbf{z}_{s}=\mathbf{z}_{s}\right)\end{array}$ & \begin{tabular}{|l}
0.6625 \\
0.6343
\end{tabular} & $\begin{array}{c}0.6868 \\
0.6587\end{array}$ \\
\hline Separate Training [22] & 0.6020 & 0.6077 \\
\hline Proposed & 0.6711 & 0.6901 \\
\hline
\end{tabular}

alistically corrupted segmentations with increased uncertainty (Supple. Fig.4). These corrupted segmentations attribute to the increased model generalization (AVG Dice: 0.6709 vs 0.6901). While one may argue that characterizing and combining various image-space DAs and corruptions together could be an interesting direction to improve cross-domain performance, it is time-consuming and computationally inefficient to find the optimal DA policy [35], and has the risk of sacrificing intra-domain performance [36].

Experiment 3: Ablation study. We further investigate three key contributions: 1) the proposed targeted masking; 2) latent code decoupler $\mathcal{H}$; 3) cooperative training. Results are shown in Table 2. We can see that disabling $\mathcal{G}_{c h}, \mathcal{G}_{s p}$ drops the average Dice score from 0.6901 to 0.6584 , highlighting the effectiveness of targeted masking. Fig. 3 shows that targeted masking focuses more on attacking cardiac structures, resulting in more challenging images with mixed artifacts and under or over-segmented predictions. We compared the proposed network architecture to its two variants, where $\mathbf{z}_{i}$ and $\mathbf{z}_{s}$ are shared in two different ways. Both variants lead to inferior performance. This suggests the benefit of $\mathcal{H}$ for a more sparse $\mathbf{z}_{s}$ code. Image reconstruction requires low-level information, whereas image segmentation relies on more concentrated high-level information. Introducing $\mathcal{H}$ explicitly defines a hierarchical feature structure to improve model generalization. Lastly, we compared our method to the state-of-the-art denoising auto-encoder-based shape refinement method (Separate Training) 22] where 
FTN and STN are trained independently. It has been shown that this learningbased method can outperform the commonly used non-learning-based condition random field-based refinement method 37]. Results show that our method can greatly outperform this advanced method by a large margin (Dice score 0.6901 vs. 0.6077), highlighting the benefits of the cooperative training strategy for enhancing learning-based shape refinement and correction.

\section{Conclusion}

We present a novel cooperative training framework in together with a latent space masking-based DA method. Experiments show that it greatly improves model generalizability and robustness against unforeseen domain shifts. Unlike existing methods which require multi-domain datasets or domain knowledge to specify particular forms of image transformation and corruption functions, our latent space DA method requires little human effort, and it has the potential to be applied to other data-driven applications. Although we only demonstrate the performance for cardiac image segmentation, our generic framework has the potential to be extended to a wide range of data-driven applications.

Acknowledgment: This work was supported by the SmartHeart EPSRC Programme Grant(EP/P001009/1).

\section{References}

1. Dinggang Shen et al. Deep learning in medical image analysis. Annual review of biomedical engineering, 19:221-248, June 2017.

2. Geert Litjens et al. A survey on deep learning in medical image analysis. Medical image analysis, 42:60-88, 2017.

3. Qi Dou et al. Domain generalization via model-agnostic learning of semantic features. In Hanna M. Wallach et al., editors, NeurIPS 2019, pages 6447-6458, 2019.

4. Isabela Albuquerque et al. Improving out-of-distribution generalization via multitask self-supervised pretraining. arXiv preprint arXiv:2003.13525, 2020.

5. Prithvijit Chattopadhyay et al. Learning to balance specificity and invariance for in and out of domain generalization. In European Conference on Computer Vision, pages 301-318. Springer, 2020.

6. Shujun Wang et al. Learning from extrinsic and intrinsic supervisions for domain generalization. In $E C C V, 2020$.

7. Shiv Shankar et al. Generalizing across domains via cross-gradient training. In ICLR. OpenReview.net, 2018.

8. Kahneman Daniel. Thinking, fast and slow, 2017.

9. Connor Shorten et al. A survey on image data augmentation for deep learning. Journal of Big Data, 6(1):60, July 2019.

10. Ling Zhang et al. Generalizing deep learning for medical image segmentation to unseen domains via deep stacked transformation. IEEE Trans. Medical Imaging, 39(7):2531-2540, 2020. 
11. Chen Chen et al. Improving the generalizability of convolutional neural networkbased segmentation on cmr images. Frontiers in cardiovascular medicine, 7:105, 2020.

12. Terrance Devries et al. Improved regularization of convolutional neural networks with cutout. CoRR, abs/1708.04552, 2017.

13. Raphael Gontijo Lopes et al. Improving robustness without sacrificing accuracy with patch gaussian augmentation. CoRR, abs/1906.02611, 2019.

14. Zongwei Zhou et al. Models genesis: Generic autodidactic models for 3d medical image analysis. In MICCAI 2019, pages 384-393, Cham, 2019. Springer International Publishing.

15. Zongwei Zhou et al. Models genesis. Medical Image Analysis, 67:101840, 2021.

16. Takeru Miyato, Shin-Ichi Maeda, Masanori Koyama, and Shin Ishii. Virtual adversarial training: A regularization method for supervised and Semi-Supervised learning. TPAMI, 2018.

17. Chen Chen et al. Realistic adversarial data augmentation for mr image segmentation. In MICCAI, pages 667-677. Springer, 2020.

18. X. Zhang et al. Deep adversarial data augmentation for extremely low data regimes. IEEE Transactions on Circuits and Systems for Video Technology, 31(1):15-28, 2021.

19. Long Zhao et al. Maximum-entropy adversarial data augmentation for improved generalization and robustness. In NeurIPS, 2020.

20. Wenzhao Zheng, Zhaodong Chen, Jiwen Lu, and Jie Zhou. Hardness-aware deep metric learning. In $C V P R$, pages $72-81,2019$.

21. Yu Zhang et al. A survey on multi-task learning. arXiv preprint arXiv:170\%.08114, 2017.

22. Agostina J Larrazabal et al. Anatomical priors for image segmentation via postprocessing with denoising autoencoders. In MICCAI 2019, pages 585-593. Springer International Publishing, 2019.

23. Nathan Painchaud et al. Cardiac mri segmentation with strong anatomical guarantees. In MICCAI, pages 632-640. Springer, 2019.

24. Naftali Tishby et al. The information bottleneck method. arXiv preprint physics/000405\%, 2000.

25. Zeyi Huang et al. Self-challenging improves cross-domain generalization. In Andrea Vedaldi, Horst Bischof, Thomas Brox, and Jan-Michael Frahm, editors, ECCV, volume 12347 of Lecture Notes in Computer Science, pages 124-140. Springer, 2020.

26. Jonathan Tompson et al. Efficient object localization using convolutional networks. In $C V P R$, pages 648-656. IEEE Computer Society, 2015.

27. Aleksander Madry, Aleksandar Makelov, Ludwig Schmidt, Dimitris Tsipras, and Adrian Vladu. Towards deep learning models resistant to adversarial attacks. In ICLR, June 2017.

28. Ian J Goodfellow et al. Explaining and harnessing adversarial examples. In $I C L R$, 2015.

29. Olivier Bernard et al. Deep learning techniques for automatic MRI cardiac Multi-Structures segmentation and diagnosis: Is the problem solved? TMI, 0062(11):2514-2525, November 2018.

30. Víctor M. Campello et al. Multi-centre, multi-vendor and multi-disease cardiac segmentation: The m\&ms challenge. ieee transactions on medical imaging, under review. TMI. 
31. Fernando Pérez-García et al. TorchIO: a Python library for efficient loading, preprocessing, augmentation and patch-based sampling of medical images in deep learning. arXiv:2003.04696 [cs, eess, stat], March 2020.

32. Olaf Ronneberger et al. U-Net: Convolutional networks for biomedical image segmentation. In MICCAI 2015, volume 9351 of Lecture Notes in Computer Science, pages 234-241. Cham, May 2015.

33. Zhenlin $\mathrm{Xu}$ et al. Robust and generalizable visual representation learning via random convolutions. In $I C L R, 2021$.

34. Terrance Devries et al. Improved regularization of convolutional neural networks with cutout. CoRR, abs/1708.04552, 2017.

35. Ekin D Cubuk et al. Autoaugment: Learning augmentation strategies from data. In $C V P R$, pages 113-123, 2019.

36. Richard Shaw et al. Mri k-space motion artefact augmentation: Model robustness and task-specific uncertainty. In M. Jorge Cardoso et al., editors, MIDL, volume 102 of Proceedings of Machine Learning Research, pages 427-436, London, United Kingdom, 08-10 Jul 2019. PMLR.

37. Patrick Ferdinand Christ et al. Automatic liver and lesion segmentation in CT using cascaded fully convolutional neural networks and $3 \mathrm{D}$ conditional random fields. In MICCAI 2016, pages 415-423. Springer International Publishing, 2016. 


\section{A Supplementary Material}

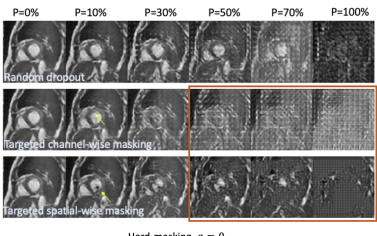

(a) Corrupted images

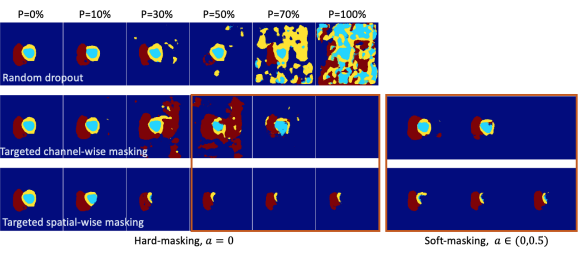

(b) Corrupted segmentations

Supple. Figure 1: Three types of latent code masking schemes generate (a) a diverse set of challenging images with unseen mixed artifacts, e.g. 'dark dots', 'checkerboard artifacts', 'blurring' and (b) various over-segmented and undersegmented predictions at different thresholds $p$. Compared to hard-masking, softmasking produces milder but more diverse corrupted images and segmentation maps. a: the annealing factor for soft masking.

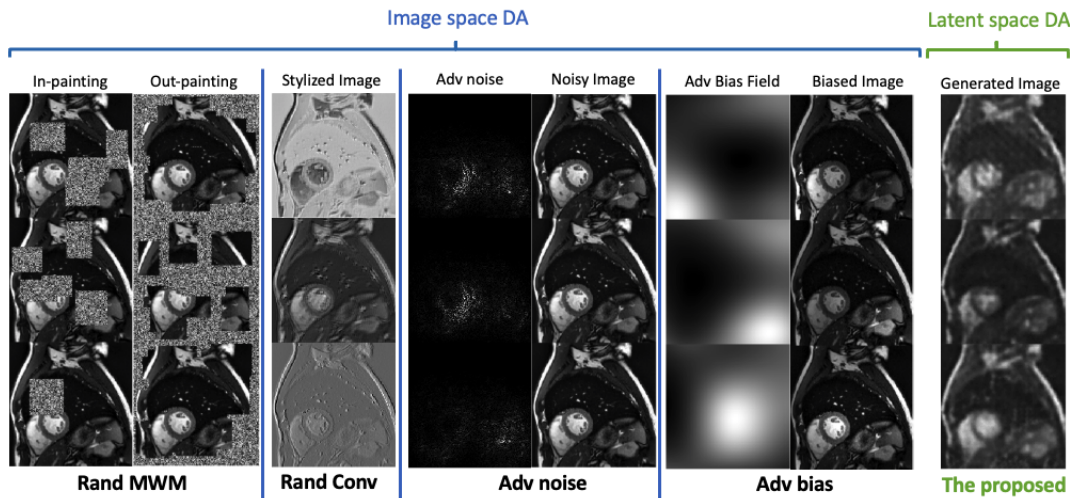

Supple. Figure 2: Visualization of input space data augmentation and latent space data augmentation (ours). DA: data augmentation. Adv: Adversarial. 


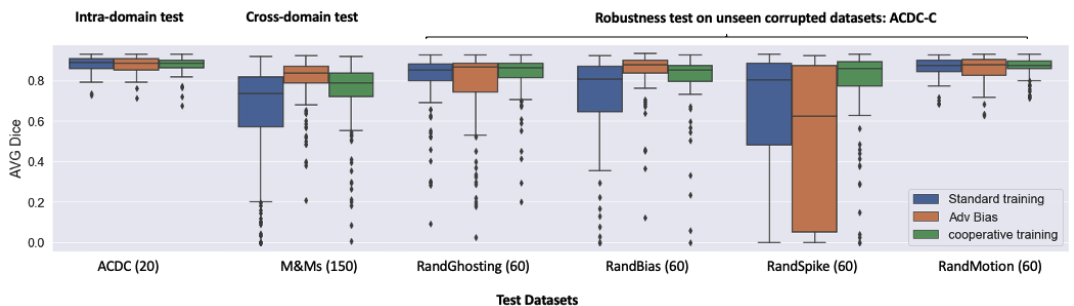

Supple. Figure 3: In the large training data setting (70 ACDC subjects), when compared to the baseline method (standard training), our cooperative training method can further improve not only intra-domain segmentation accuracy (with reduced variance) but also robustness against various domain shifts. Adv bias, by contrast, fails to provide consistent improvement. This reveals our method's great potential to be applied to a wide range of scenarios for both improved generalization and robustness.
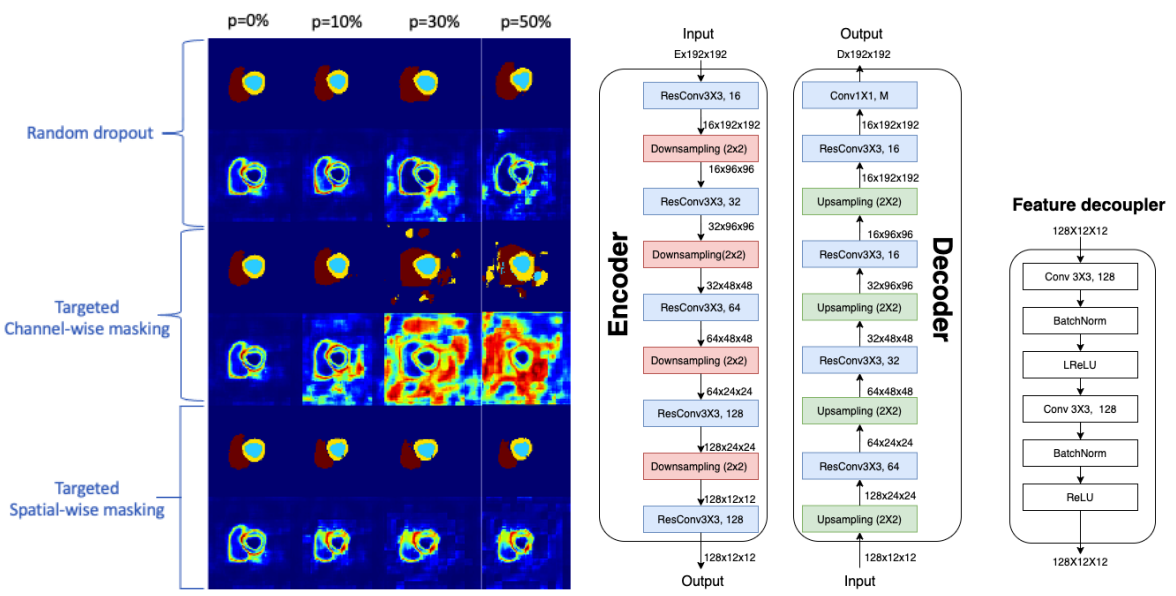

Supple. Figure 4: Visualization of cor- Supple. Figure 5: Structures of Unetrupted segmentation (the first row in each block) and the corresponding entropy map (the second row in each block) using the proposed three latent space masking schemes. Latent masking schemes generate realistic poor segmentation with increased entropy, which is beneficial to train our denoising autoencoder (STN) for shape correction. like encoder-decoder pairs, and the feature decoupler used in this paper. We use the same structures for encoders and decoders accordingly. E: \# of input channel(s), D: \# of output channel(s). ResConv: Convolutional Block with residual connections [?]. Conv: Standard convolutional kernels. Of note, our framework is generic, other encoders and decoders can also be used. 